\title{
Brainstorming 2.0: Toward collaborative tool based on social networks
}

\author{
MohamedChrayah ${ }^{1}$,Kamal Eddine El Kadiri ${ }^{1}$, Boubker Sbihi ${ }^{1}$,Noura Aknin ${ }^{2}$ \\ ${ }^{1}$ Laboratory LIROSA, ${ }^{2}$ Laboratory LaSIT \\ Faculty of sciences \\ Tétouan, Morocco
}

\begin{abstract}
Social networks are part of Web 2.0 collaborative tools that have a major impact in enriching the sharing and communication enabling a maximum of collaboration and innovation globally between web users. It is in this context that this article is positioned to be part of a series of scientific research conducted by our research team and that mixes social networks and collaborative decision making on the net. It aims to provide a new tool open source for solving various social problems posed by users in a collaborative $\mathbf{2 . 0}$ based on the technique for generating ideas, brainstorming method and social networks together for the maximum possible adequate profiles to the virtual brainstorming session. A tool is run by a user called expert accompanied by a number of users called validators to drive the process of extracting ideas to the loan of various users of the net. It offers then the solution to the problem of sending a satisfaction questionnaire administered by an expert ready for the affected user to measure the level of his satisfaction and also the success of the process launched. For its implementation, we propose a unified modeling using UML language, followed by a realization using the JAVA language.
\end{abstract}

Keywords- component: Web2.0, brainstorming, social networks, UML.

\section{INTRODUCTION}

Over time, the web has seen many changes starting with the static web which allowed only to display static pages made by the directors of the net and whose content was not always updated followed by collaborative Web 2.0 who proposed the involvement of users in content creation. Web 2.0 was proposed in August 2001 by Dale Dougherty of O'Reilly Media, but the real release of concept 2.0 was published in an international conference in 2005 by Tim O'Reilly [10]. He proposed a new vision of the web which consists on a higher participation of Internet users as producers of information thus forming communities participating in the communication, sharing and dissemination of information.

With this concept a lot of software and services are freely available on the web and therefore the amount of information has increased which encouraged users to participate and inter exchange. Social networks have existed since 2003, where they have grown exponentially up to date [7]. They collect data on members, and then store this information as data profiles, these sites represent an appropriate database to search for suitable profiles to any operation or survey in the web. Moreover, decision-making has changed a lot with the emergence of information and communication technologies (ICT) [6]. Makers become less statically located; on the contrary, they act in a distributed manner. This change creates a new set of requirements: collaborative decision-making based on collaboration using Web 2.0 tools.

In the next section, we present the web 2.0; its principles, its most used tools and especially social networking the tool used in our article, and then we'll present the notion of collaborative decision based on the method of generating ideas (brainstorming) and social networks to achieve in the end a collaborative decision as a result of a series of proposals and virtual meeting by web users 2.0. Finally, we'll propose a design and implementation of the proposed tool using the UML.

\section{WEB 2.0}

\section{A. Web 2.0 and its dimensions}

Web 2.0 is social, is open, it lets you control your data, mixing the global with the local. Web 2.0 is new interfaces new ways to search and access content. Web 2.0 is a platform ready to receive the educators, the media, politics, and communities. Thus, users who contribute to information exchange can simply interact (share, exchange, etc.) with both the content and page structure, but also between them, including creating the social Web. The user becomes, using the tools at its disposal, an active person of the cloth [9].

Web 2.0 can be viewed in three dimensions as shown in the following figure:

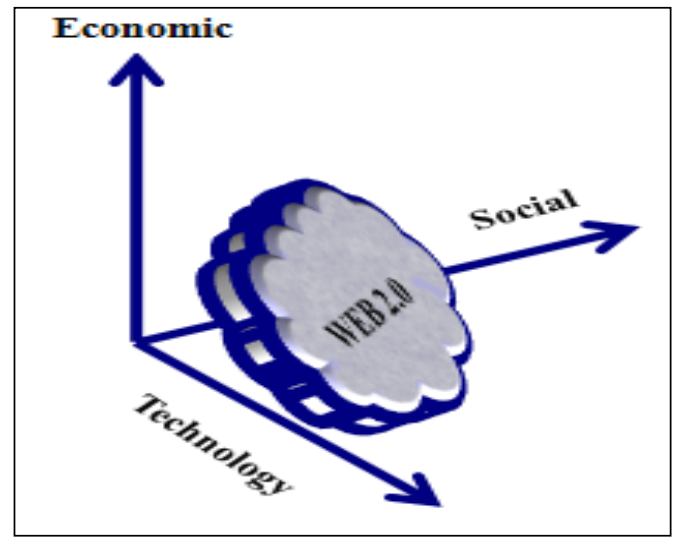

Figure 1: Dimensions Of Web 2.0. 
Social dimension: Web 2.0 is a real network of social interaction based on the participation of Internet users. User communities are created in this context based on areas of mutual benefit. Anyone can easily create an information space accessible by anyone and anywhere in the world where he can put anything (anything is the one of the boundaries of Web 2.0).

Technical dimension: Web 2.0 is an advanced technique that makes it simple to access the production and use of information through the tilting of the software installation to use online services. Thanks to the use of multiple technologies (XHTML, CSS and JavaScript for the presentation of the sites, DOM, Document Object Model, for dynamic and interactive signage, XML and XSLT for data manipulation).

Economic dimension: Funding Web 2.0 sites is done mainly through advertisements, commercial offers and trafficking networks instead of gifts or payments for licenses to use proprietary software. A project based on an economic model of Web 2.0 is based on the large mass of users who consume information mixed with advertising or commercial content which finances containers [2].

\section{B. The Tools Of Web 2.0}

Web 2.0 consists of a set of Internet technologies that facilitate the open and participatory work. Its main feature is that Web 2.0 tools allow users to control the network and interact proactively to improve or transform situations that affect them.

\section{1) Blog:}

The term "blog" is short for weblog, which can be translated as "Internet newspaper". Frequently defined as a personal site, this is an individual space of expression, created to give voice to all Internet users (individuals, businesses, artists, politicians, associations ...). Blogs are extremely simplified sites and dedicated to writing, where "the entries appear in ante-chronological order." The animation of blogs is initially limited to technophiles capable of creating the structure of their blog and have it hosted on a server.

The Pew Research Center's Internet and American Life Project has conducted a survey in 2008 which has resulted in startling statistics that $40 \%$ of adult Internet users in the United States have blogs.

Blogs have been discussed recently as a innovative knowledge of sharing technology, knowledge and management [12].

\section{2) Wiki:}

The term is derived from the expression Hawaiian wikiwiki, meaning quick. A wiki is a collaborative website whose content can be edited by visitors on the site, allowing users to easily create and edit collaborative web pages [8]. In essence, a wiki is a simplification of the process of creating HTML web pages combined with a system that records each individual change that occurs over time, so that at any time a page can be forced to return to the one of its previous states. A wiki can also provide tools that allow the users community to monitor the changing state of the wiki and discuss issues that arise. Some wikis restrict access to a group of members, allowing only members to change the page content but everyone can see it. Others allow unrestricted access, allowing anyone to both modify the content and display.

3) Social Networks:

A social network is a set of social entities such as individuals or social organizations linked together by bonds created during social interactions.

It is represented by a structure or a dynamic form of a social group it's a web space to:

- Express them selves

- To promote itself

- Exchange

- Get back in touch

Social networks have as common basis the sharing:

- Sharing knowledge

- Sharing of professional contacts

- Content Sharing

Social networks are social websites that enable people to form online communities and share content created by these users. People can be users of the open Internet or restricted to those who belong to a particular organization (eg company, university, etc.). [14]

Table 1 gives an idea of the number of users of social networks, and the classification of these social sites depending on the number of participants.

\section{TABLE I. SOCIAL NETWORKS USED MOST [15]}

\begin{tabular}{|l|c|}
\hline \multicolumn{1}{|c|}{ Site Name } & Users (in Million) \\
\hline Facebook & 309 \\
\hline MySpace & 253 \\
\hline WindowsLiveSpaces & 120 \\
\hline Habbo & 117 \\
\hline Friendster & 90 \\
\hline Hi5 & 80 \\
\hline Tagged & 70 \\
\hline Orkut & 67 \\
\hline Flixter & 63 \\
\hline
\end{tabular}

As the table shows, Facebook is positioning itself in first place with 309 million users. The implication of this number in a decision-making would be a dream for designers of collaborative Web 2.0

4) RSS Feeds:

RSS (Really Simple Syndication) is a simple XML syntax to describe the recent additions of content to a website. These additions can include elements of news, blog updates, library acquisitions or any other information. it just facilitates dynamic sharing of content between a publisher (website) and a reader (the Internet) by allowing authors and editors of a website to make available to the community some content that can be reused for integration into another site [13]. Since RSS uses XML to disseminate information relevant to user needs, RSS could well become the universal method for extracting information from the Internet. 


\section{BRAINSTORMING 2.0:COLLABORATIVE DECISION MAKING}

\section{A. Brainstorming Method:}

Brainstorming is a technique for generating ideas that stimulates creative thinking in finding solutions to a given problem. This is to produce as many ideas as possible in the shortest time on a given topic without criticism, without judgment. This searching method favors quantity, spontaneity and imagination [11].

Table II shows the essential steps for a successful brainstorming session:

\section{TABLE II. STEPS BRAINSTORMING}

\begin{tabular}{|c|l|}
\hline Steps & \multicolumn{1}{|c|}{ Sequence } \\
\hline Step 1 & Presentation of the problem \\
\hline Step 2 & warming period \\
\hline Step 3 & Brainstorming \\
\hline Step 4 & classification / grouping of ideas \\
\hline Step 5 & Final Decision \\
\hline
\end{tabular}

As shown in Figure 3, the Collaborative Decision Making Brainstorming consists of four iterative steps starting with the presentation of the problem and ending with the classification of ideas.

\section{B. Brainstorming2.0:}

1) Towards a tool for collaborative decision making:

The collaborative decision making is based on user's participation as actors for the production and wide dissemination of the decision subsequently forming communities. The size and mass of decisions will increase the quantitative level but still the qualitative decisions suggested by users.

The users of the system must be ordered according to their importance and give more privileges. It is not enough to give any decision, but the right decision, for this we proposed Brainstorming 2.0 tool to overcome the problems already discussed above.

Brainstorming 2.0 is an Open Source, free dedicated to all users of Web 2.0; their goal is to found a topic concerning decisions. It is not as tools publisher's social owner as Google Plus (G+), which is a social network where there are paid services, and their only purpose the meeting between friend or the professional. Brainstorming 2.0 is a social tool that organizes virtual brainstorming sessions between users of the web communities.

\section{2) Classification Decisions:}

The decision generated in the brainstorming 2.0 system can be classified into four classes [4][3][5][1] according to their quality:
TABLE III. CALASSIFICATION DECISIONS

\begin{tabular}{|c|c|c|}
\hline Code & libel & Weighting \\
\hline G & Good & 10 \\
\hline M & Meduim & 5 \\
\hline L & Low & 1 \\
\hline E & Error & -10 \\
\hline
\end{tabular}

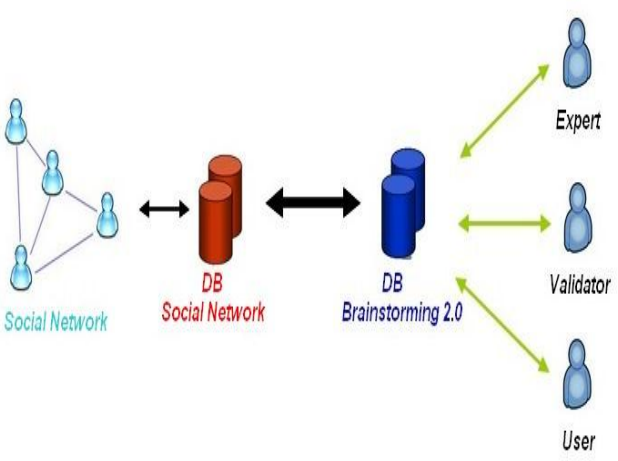

Figure2. Brainstorming 2.0 Architecture

According to the above table the user who produces the right decisions and averages has the chance to become a validator in a short time; it also proposes to create a virtual currency that will increase every time someone publishes validated decisions. Users will then have access to some opportunity not given to all others. This value will depend on the turnout of participation in the virtual brainstorming sessions and also the notation affected by the validators.

\section{3) Classification Of Users:}

It is proposed to decompose the system users into three groups: simple users who consume and produce decisions, the validators who validate decisions and finally the experts who make tracking validations, pointing validators for each problem and its publication when validated by the validators.

A simple user can become a validator if its weight exceeds $1000 \mathrm{pt}$ and is recommended by an expert, a validator must communicate with other validators and expert in the validation process. A validator can become an expert if its weight exceeds 10000 pt. Brainstorming 2.0 users are represented in the following table [4][3][5][1]:

TABLE IV. Classement Des Utilisateurs

\begin{tabular}{|c|c|c|}
\hline Code & Libel & Weighting \\
\hline E & EXPERT & $>=10000$ \\
\hline V & VALIDATOR & $>10000$ And $>=1000$ \\
\hline U & USER & $<=1000$ \\
\hline
\end{tabular}

Regarding the weighting at each decision by a user there is an increase in the value using the following formula (1): 


\section{$P($ User $)=\Sigma P($ Decisions $)$}

(1)

The following figure shows the use case diagram tool braistorming 2.0

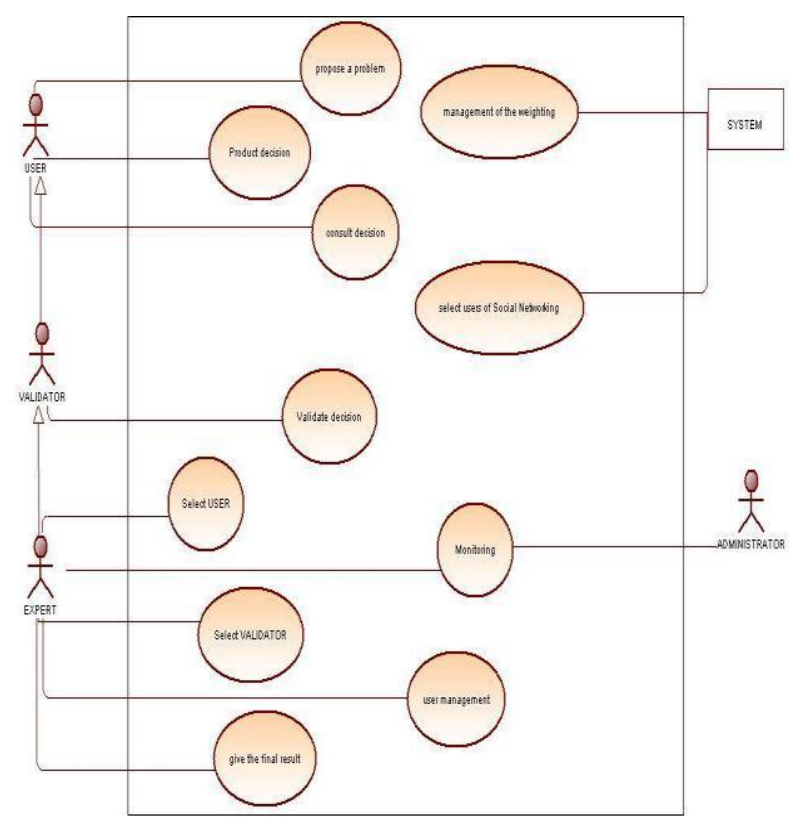

Figure3 Uses Cases Brainstorming 2.0.

The use case diagram shown in Fig.3 clarified the interaction between the system and all actors (users), it is clear from the diagram that the expert is the main user of the system, he inherits all the actions of other users in addition to managing the flow of information he can administrate the platform. The actor system is who is responsible to the updating of weights and linking with social networking to select the appropriate profiles.

\section{4) Decision Making Process In The 2.0 Brainstorming:}

The process of decision making in the Brainstorming 2.0 runs as follows, figure 4 .

A user poses a problem, a system expert considers the issue and distributes it to all users with an adequate profile to this subject by searching social networks related to the system, and in parallel the expert selects validators to validate decisions proposed by them. After treatment and decisions classification by the validators, the expert groups the decisions that have obtained Class B (Good) by the validators to generate a final decision of the issue to be released in the portal. Finally a satisfaction questionnaire is sent to the user who submitted the problem to get an idea about his satisfaction with the solution proposed.

The figure. 5 gives us an idea about the chronological interactions between all major and minor players in the system, from the diagram it is the simple user who initiates the transaction, the one who started the virtual brainstorming session, then after the analysis of the issue the expert send it to other users to give these decisions and in the end transfer them to the validators.

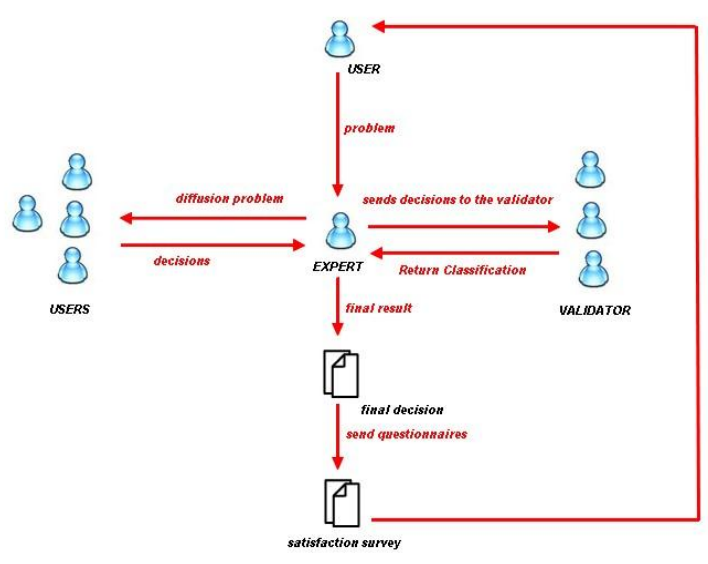

Figure4 Process Brainstorming 2.0.

But the sequence diagram is insufficient to give us an overview of the system, that is why we use the class diagram to understand the relationship between the classes of the system, the Fig6 represents the class diagram of the brainstorming 2.0 system; According to the diagram the system is composed of 9 classes, Expert class inherits the methods of the Validator class that also inherits from the User class, the class Profil_Network_Social contains information on existing profiles in social networks interacting with the system.

It is clear that the class Decision_final is related directly to the Expert class as the one who distributes the final decision, Decision_Validate class with the class validator and finally the classes Decision and Problem with the USER class, because he's the one who launches the problem and also the one who gives decisions.

\section{EXAMPLE : A CASE STUdy.}

An expert receives a user's question "What is your opinion on Web 2.0?" And then he launches it into the system, after consultation with user's six users answered the question with judgment:

- User1 : Favourable opinion

- User2 : Unfavourable opinion

- User3 : Favourable opinion

- User4 : Favourable opinion

- User5 : Unfavourable opinion

- User6 : Favourable opinion 


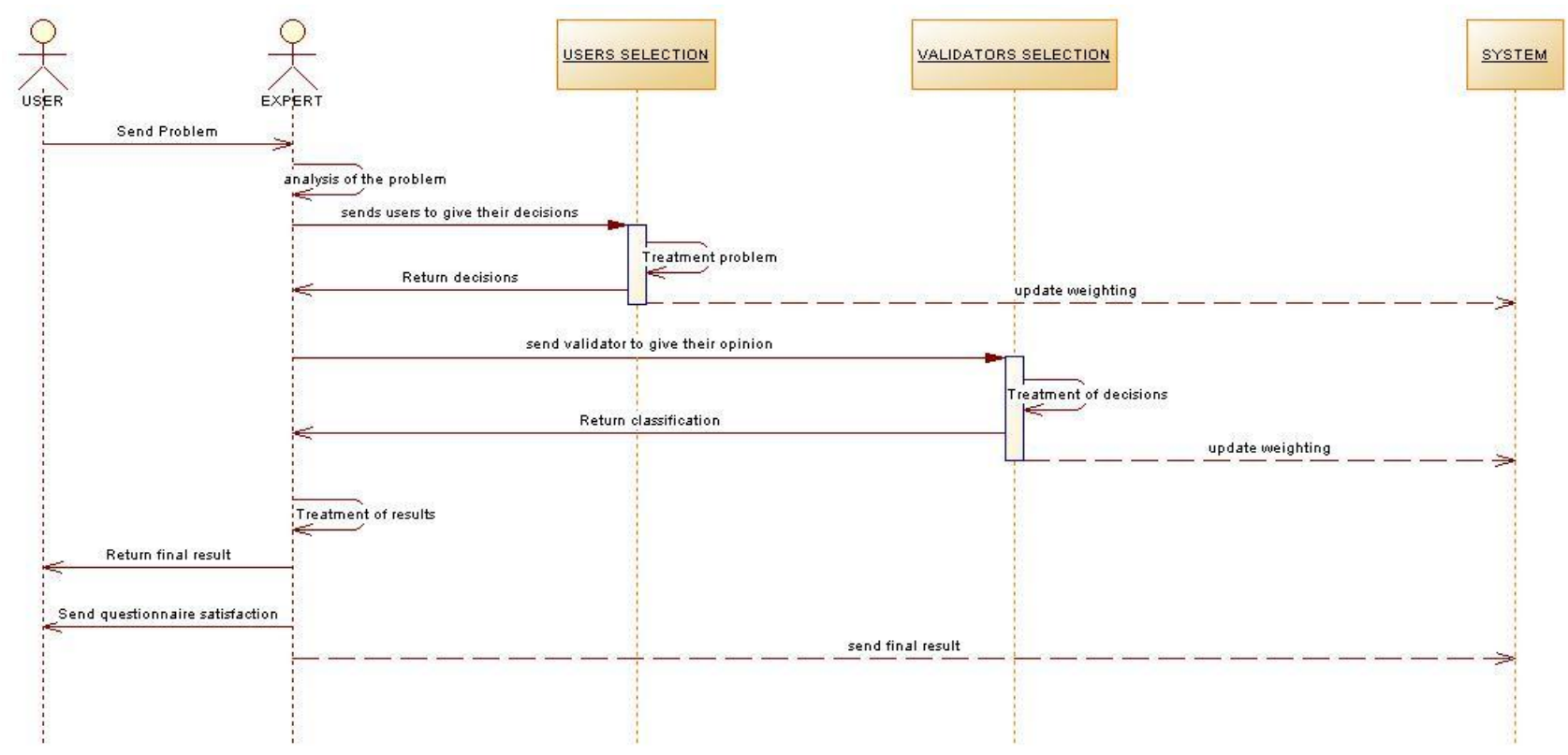

Figure5 Sequence Brainstorming 2.0 Diagram

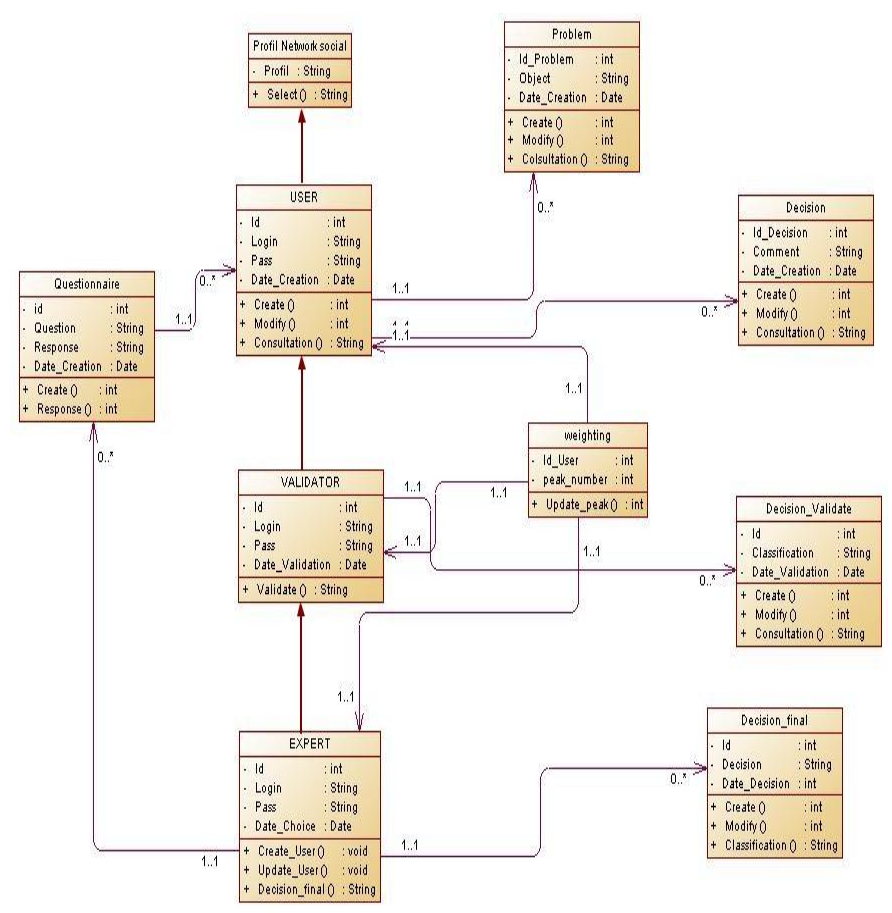

Figure6 Class Brainstorming 2.0 Diagram

The expert selects two system validators to review and validate user responses by giving them a ranking:

TABLE V. RANKING ANSWERS

\begin{tabular}{|c|c|c|}
\hline & Validator1 & Validator2 \\
\hline User1 & G & G \\
\hline User2 & L & L \\
\hline User3 & G & G \\
\hline
\end{tabular}

\begin{tabular}{|c|c|c|}
\hline & Validator1 & Validator2 \\
\hline User4 & G & G \\
\hline User5 & L & L \\
\hline User6 & G & G \\
\hline
\end{tabular}

After ranking the responses, the expert selects the response that received a Class B by both validators and diffuses it on the portal (Favourable Opinion) with the judgment of users who responded favorably, at the end the expert sends a questionnaire to the user that sent the question to get an idea of his level of satisfaction with the answer.If the user is satisfied, it will make him seek help and become part of the community of this tool and if it is not satisfied, he can restart another process or ask for help from People experts in the wanted domain. What is clear is that decisions vary from personal context to professional staff. To improve performance in a professional context, it might be thought to pay employees and to create training sessions to achieve meaningful results at the expense of a sum of money to take advantage of collaborative network intelligence.

\section{CONCLUSION}

The aim of our proposal is to find a mechanism mixing between the benefits of Web 2.0 tools and the technique for generating ideas brainstorming in order to achieve a system of collaborative decision making. This new system will help find lot of solutions through their connection with social networks, which contains adequate profiles and also good decisions because the raw information has no value in the new system and as the one who adopts the problem is an expert. The limitations of this tool are that it contradicts the general concept of Web 2.0 (the participation of everyone in the decision) since the expert takes some decision. There is also the responsibility of the validators in the selection and classification decisions. In addition, it is necessary to test and 
measure the level of satisfaction undefined users to meet the needs of each context.

This article is a beginning of a series of articles that will follow and that will be the implementation of this tool in java. A presentation detailed the tool will be made in future publications

\section{PERSPECTIVE}

As prospects we propose to generalize the use of this tool and measure the rate of satisfaction of its users. We propose also to design tools for collaborative semantic decision support which understands the sense of the decision. Adapt this tool to other collaborative tools are blogs, wikis and RSS feeds. Impose a single and secure identification with fingerprints to ensure good use away from hackers and malicious people.

\section{REFERENCES}

[1] Boubker Sbihi, K.E. El Kadiri, and N. Aknin, "The Vblogs: Towards a New Generation of Blogs", International Journal of Computer Science Issues, Vol. 7, issue 3, No. 2, May 2010, pp. 9-14.

[2] Boubker Sbihi, TOWARDS A NEW VISION OF WEB 2.0, Computer Sciences and Telecommunications, 2009

[3] Boubker Sbihi, and Kamal Eddine El Kadiri, "Web 2.2: Toward classified information on the Web", International Journal of Web Applications, Vol. 1, No. 2, June 2009, pp. 102- 109

[4] Chrayah Mohamed, Kamal Eddine El Kadiri, Boubker Sbihi and Noura Aknin, Brainstorming +: Towards a tool for decision-making In the Web 2.0, IJCSI International Journal of Computer Science Issues, Vol. 9, Issue 2, No 2, March 2012

[5] Chrayah Mohamed, Boubker Sbihi and Kamal Eddine El Kadiri :Towards an instrument for a new generation of blog. EMSCE 2011 May 19202011 ISBN:978-84-694-4025-4.

[6] Claus Rinnera,Carsten Keßlerb,Stephen Andrulisa, The use of Web2.0 concepts to support deliberation in spatial decision-making, Computers, Environment and Urban SystemsVolume 32, Issue 5, September 2008, Pages 386-395.
[7] Danah boyd, Nicole Ellison, Social Network Sites: Definition, History, and ScholarshipJournal of Computer-Mediated Communication Volume 13, Issue 1, pages 210-230, October 2007.

[8] Howard T. Welser ,Patrick Underwood, Wiki Networks: Connections of Creativity and Collaboration 2011, Pages 247-271.

[9] Musser J, O'Reilly T. O'Reilly Radar Report: Web 2.0 Principles and Best Practices: O'Reilly Media 2006.

[10] O’Reilly T. What Is Web 2.0? Design Patterns and Business Models

[11] for the Next Generation of Software; 2005.

[12] Patrick C.Shih, Gina Venolia, Gary M. Olson Brainstorming under constraints: Why software developers brainstorm in groups 2011 Pages 74-83

[13] Sangmi Chaia, Minkyun Kimb, What makes bloggers share knowledge? An investigation on the role of trust, International Journal of Information Management 30 (2010) 408-415

[14] Yu-Feng Lan,Yang-Siang Sie, Using RSS to support mobile learning based on media richness theory Volume 55, Issue 2, September 2010, Pages 723-732

[15] WonKim a, Ok-RanJeong a, Sang-WonLee On social Web sites Information Systems 35 (2010) 215-236

\section{AUTHORS PROFILE}

Chrayah Mohamedis computer engineer, pHD student and themember of LIROSA laboratory.

Kamal Eddine El Kadiriis pHD doctor and professor of computerscience at Faculty of Sciences of Tétouan in Morocco. He is theDirector of the ENSA School of engineers of Tetouan and theDirector of LIROSA laboratory. He has published several articleson E-learning and Web 2.0. He is part of many boards ofinternational journals and international conferences.

BoubkerSbihiis pHD doctor and professor of computer scienceat the School of Information Science in morocco. He is theresponsible of Department of Information Management. He haspublished many articles on E-learning and Web 2.0. He is part ofmany boards of international journals and international conferences.

NouraAkninis pHD doctor and professor of computer science atFaculty of Sciences of Tétouan in Morocco. She has publishedmany articles on Elearning and Web 2.0. She is part of manyboards of international journals and international conferences. Shehas member of the IEEE and the IEEE Computer Society 